\title{
Servicios para la atención y la prevención de defectos congénitos. Síntesis de una reunión de la O rganización Mundial de la Salud y la Fundación March of Dimes
}

\author{
Silvia Castillo Taucher ${ }^{1}$. \\ Services for the care and prevention \\ of birth defects. Reduced report \\ of a World Health O rganization and \\ March of Dimes Foundation meeting
}

On 17-19 May 2006, the World Health Organization (WHO) and the March of Dimes Birth Defects Foundation held a meeting in Geneva: The Management of Birth Defects and Haemoglobin Disorders. Meeting participants included 18 experts from developing and industrialized countries, including the author and nine staff from WHO Headquarters. The meeting had five goals: (A) ratify the data on the global toll of birth defects presented in the MOD Global Report; (B) agree upon a definition of terms; (C) develop a collaborative plan for strengthening care and prevention of birth defects; (D) develop a plan for strengthening care and prevention of haemoglobin disorders; and (E) determine how potential stakeholders could contribute to these efforts. The consensus for each of the goals were: a) Participants endorsed the estimates in the MOD Global Report. b) Participants concluded that the term "birth defect" is synonymous with the term "congenital disorder", whereas the term "congenital anomalies" should be avoided. c) Participants agreed that $70 \%$ of birth defects could be prevented, ameliorated or treated effectively, by the strengthening of medical genetic services. d) Participants agreed that efforts must be made to improve the control of hemoglobin disorders in developing countries. and e) Progress will require the combined efforts and political will of the WHO (Rev Méd Chile 2007; 135: 806-13).

(Key w ords: Abnormalities; Hemoglobinopathies; World Health Organization)

Recibido el 20 de octubre, 2006. Aceptado el 12 de diciembre, 2006.

Adaptación, reducción y traducción de un documento elaborado por Christopher P. Howson y Arnold Christianson. Publicación autorizada por Victor Boulyjenkov, Director de los Programas «Human Genetics»y «Chronic Diseases and Health Promotion»de la Organización Mundial de la Salud.

${ }^{1}$ Sección Genética, Departamento de Medicina, Hospital Clínico Universidad de Chile. Santiago, Chile.

Correspondencia a: Dra. Silvia Castillo Taucher. Sección

Genética, Departamento de Medicina, Hospital Clínico Uni-

versidad de Chile. Santiago, Chile. Fax: 5629788513.

E mail: scastill@redclinicauchile.cl 


\section{ANTECEDENTES}

La Organización Mundial de la Salud (OMS) y la Fundación March of Dimes (MOD), entre 1950 y 1960, reconocieron que la transición de salud en naciones industrializadas llevaría a la necesidad de desarrollar servicios médicos genéticos ${ }^{1-3}$. Como consecuencia del desarrollo científico y tecnológico, la práctica médica genética, individual o familiar, evolucionó primariamente en centros médicos terciarios y académicos ${ }^{4,5}$.

\section{ROL DE LA OMS}

En 1985, un comité asesor del Programa de Enfermedades Hereditarias, más tarde el Programa de Genética Humana (HGN), anticipó que la transición de salud en las naciones en desamollo requerinía de servicios médicos genéticos ${ }^{6}$. Basado en experiencias ganadas en estos países con la implementación de servicios médicos para las hemoglobinopatías, se reconoció que dichos servicios serían diferentes de aquellos de las naciones industrializadas. El modelo de servicios académicos y de cuidados terciarios en naciones industrializadas necesitaría ser traducido a una estrategia más holística basada en la comunidad, incorporando programas de salud pública aplicados a la atención primaria y ligados a servicios secundarios y terciarios ${ }^{6-9}$.

\section{Rol de la Fundación March of Dimes}

La Fundación March of Dimes (MOD), una organización no gubernamental en los Estados Unidos de Norteamérica, fue creada en 1938, durante el mandato del Presidente Franklin D. Roosevelt, para combatir la poliomielitis. En 1958, la MOD redireccionó su misión, que actualmente consiste en mejorar la salud de los recién nacidos mediante la prevención de defectos del nacimiento, de nacimientos prematuros y de la mortalidad infantil. En 1998, la Fundación MOD de Defectos del Nacimiento extendió su misión internacionalmente a través de la creación de sus Programas Globales ${ }^{1}$.

El siguiente documento resume un informe elaborado en la reunión OMS-MOD realizada en mayo de 2006, en Ginebra, Suiza, en la que participó su autora.
OBJETIVOS DE LA REUNIÓN

Meta 1

Revisar los datos sobre la carga global de defectos del nacimiento presentado en el Reporte Global de 2006 de la MOD sobre defectos del nacimiento. El Reporte Global de 2006 de la MOD sobre defectos del nacimiento estima que 7,9 millones de niños nacen anualmente con un serio defecto genético. De estos nacimientos, el 94 por ciento $(7,4$ millones) ocurre en países de bajo y mediano ingreso. La prevalencia estimada al nacimiento de estas enfermedades oscila de 40 por 1.000 nacidos vivos, en países de alto ingreso, a un máximo de 82 por 1.000 nacidos vivos, en países de bajo ingreso. Sobre 3,3 millones de niños bajo los cinco años de edad fallecen cada año por defectos del nacimiento, y la mayoría de estas muertes ocurre en países en desarrollo ${ }^{10}$. Estas muertes, debidas directamente al defecto del nacimiento 0 como consecuencia del defecto de nacimiento, que actúa como un factor de comorbilidad, han sido largamente desconocidas.

Se apoyaron las estimaciones del Reporte Global de la MOD, y sugirieron reforzar y expandir la base de datos. Además, apoyaron las recomendaciones para la implementación y reforzamiento de servicios médicos genéticos en países en desarrollo. También reafirmaron las recomendaciones de dos reportes previos de la $\mathrm{OMS}^{7,11,12}$.

\section{Meta 2}

Acuerdo sobre la definición de términos. El desarrollo de políticas coherentes de salud y servicios apropiados, requiere datos epidemiológicos confiables sobre la carga de las enfermedades ${ }^{13}$. El primer paso es la clara definición y clasificación de las enfermedades a revisar. Ha faltado un acuerdo en la definición de enfermedades que son congénitas, esto es, existentes desde el nacimiento» 0 de etiología prenatal ${ }^{14}$.

El término preferido de la MOD es «defectos del nacimiento »y los define como anomalías en la estructura o función, incluyendo metabolismo, que están presentes desde el nacimiento. Esta definición incluye algunos defectos congénitos clínicamente obvios al nacimiento y otros que se manifiestan más tarde en la vida.

El término «defecto serio del nacimiento»es considerado por la MOD como aquél que com- 
prende enfermedades que amenazan la vida 0 pueden resultar potencialmente en discapacidad. $\mathrm{Su}$ etiología incluye enfermedades genéticas (defectos monogénicos y anomalías cromosómicas), enfermedades parcialmente genéticas (malformaciones congénitas multifactoriales); enfermedades consecuencia de factores ambientales anormales, incluyendo teratógenos que distorsionan el crecimiento y desarrollo normales del embrión o feto; fuerzas mecánicas que deforman al feto; accidentes vasculares que producen disrupción del crecimiento normal de órganos y extremidades, y causas desconocidas ${ }^{14,15}$.

El HGN prefiere el término enfermedad congénita», definida como cualquier condición potencialmente patológica aparecida antes del nacimiento, incluyendo todas las enfermedades causadas por factores ambientales, genéticos o desconocidos, ya sea que sean evidentes al nacimiento o se hagan manifiestas más tarde en la vida ${ }^{6,12}$. La OMS también ha usado los términos malformaciones congénitas, deformaciones y anomalías cromosómicas» que están en la 10a Clasificación Intemacional de Enfermedades (ICD 10) primariamente en su base anatómica, más que por etiología ${ }^{16}$. Colectivamente, estas enfermedades comprenden lo que la Unidad de Carga de Enfermedad de la OMS llama 〈enfermedades congénitas» ${ }^{7}$.

Anomalías congénitas son alteraciones morfológicas macroscópicas presentes al nacimiento. Un problema es que excluye defectos funcionales como discapacidades congénitas no sindrómicas (intelectual, física, visual, auditiva y epilepsia), enfermedades monogénicas comunes como las hemoglobinopatías, deficiencia de glucosa-6-fosfato deshidrogenasa, fibrosis quística, albinismo oculocutáneo, atrofia muscular espinal y errores innatos del metabolismo. También excluye muchos defectos del nacimiento frecuentes e inducidos por teratógenos, como sífilis congénita, rubéola congénita y deficiencia de yodo ${ }^{16,18}$. Desafortunadamente, la ICD 10 continúa siendo ampliamente usada para recolectar e intercambiar datos relacionados con defectos del nacimiento. Sus deficiencias han impulsado el desarrollo de numerosas clasificaciones alternativas y sistemas de codificación, que incluyen aquellos del Centro de Control de Enfermedades de los Estados Unidos de Norteamérica, la Asociación Pediátrica Británica y la European Concerted Action on Congenital Anomalies and Twins (EUROCAT) ${ }^{19}$.
Se concluyó que el término «defecto del nacimiento»es sinónimo con el término senfermedad congénita» y ambos pueden ser utilizados intercambiablemente.

También se alentó a la Unidad de Clasificación y Terminología de la OMS, para que en la próxima versión de la Clasificación Internacional de Enfermedades (ICD 11), se comprometa a una clasificación internacionalmente aceptable de los defectos del nacimiento.

\section{Meta 3}

Desarrollar un plan de acción colaborativa para el fortalecimiento de la atención y prevención de defectos del nacimiento en países de bajo y mediano ingreso. Se acordó que más de $70 \%$ de los defectos del nacimiento puede ser prevenido, mejorado 0 tratado efectivamente, pero en una base global requerinía del fortalecimiento de los servicios médicos genéticos en países en desarrollo, con cuidado periconcepcional, salud materna, manejo del parto, y cuidado de la salud del recién nacido y el niño con enfermedades agudas y crónicas. Los servicios para el cuidado y prevención de defectos del nacimiento también deben estar relacionados y contener programas en nutrición, inmunización, control de enfermedades infecciosas y discapacidad y rehabilitación, entre otros. Además, los servicios médicos genéticos debieran tener una fuerte base en atención primaria de salud y estar integrados con servicios de salud de atención secundaria y terciaria ${ }^{6,7,10,12,20-22}$.

Los servicios médicos genéticos debieran enfatizar la atención y la prevención. La atención incluye el reconocimiento y diagnóstico de defectos del nacimiento; el tratamiento (médico, quirúrgico y del neurodesarrollo) y consejo con apoyo psicosocial ${ }^{4,7,10}$.

Los servicios preventivos dependen de la implementación de disposiciones básicas de salud reproductiva, mejor provistas como parte del cuidado periconcepcional; incluyen planificación familiar y optimización de la salud de la mujer a través de mejorar la dieta, tratar enfermedades como diabetes mellitus insulino dependiente $\mathrm{y}$ epilepsia en forma óptima, y controlando infecciones teratogénicas. Todos los países serían capaces de proveer estos servicios ${ }^{4,7,10,12}$.

La Tabla 1 muestra las recomendaciones básicas en salud reproductiva consideradas apropia- 


\section{Tabla 1. Recomendaciones básicas en salud reproductiva consideradas apropiadas para países de bajo y mediano ingreso, para prevenir defectos del nacimiento ${ }^{23}$}

1. Promover la planificación familiar, permitiendo a las parejas espaciar los embarazos, planificar el tamaño de la familia, definir las edades a las cuales les gustaría empezar y completar sus familias y reducir la proporción de embarazos no intencionados. Esto permitiría:

- reducir la tasa total de defectos del nacimiento

- disminuir la prevalencia al nacimiento de síndrome de Down por la reducción del número de madres de edad materna avanzada

- permitir a las mujeres con hijos afectados la opción de no tener más niños

- introducir a las mujeres en el concepto de elección reproductiva

2. Asegurar una dieta sana, balanceada antes y durante los años reproductivos de la mujer a través de una ingestión adecuada de macronutrientes (proteínas, carbohidratos y grasas) y un amplio rango de micronutrientes. Esto permitiría:

- prevenir la deficiencia de yodo a través de la fortificación de la sal con yodo

- prevenir defectos del tubo neural y otras malformaciones a través de la fortificación de la harina y otros alimentos principales y por suplementación cuando se requiera

- prevenir los defectos del nacimiento debidos a teratógenos comunes como el alcohol y las drogas recreacionales

3. Controlar las infecciones en todas las mujeres antes y durante el embarazo. En particular:

- prevenir y tratar la sífilis

- prevenir el síndrome de rubéola congénita a través de la vacunación

4. Optimizar la salud materna con el control de enfermedades crónicas asociadas con riesgo aumentado de defectos del nacimiento. Blancos, en particular:

- diabetes mellitus insulino dependiente

- epilepsia y su control con drogas antiepilépticas

- mujeres con cumarínicos por trombosis de venas profundas o patología cardíaca

das para países de bajo y mediano ingreso, para prevenir defectos del nacimiento ${ }^{23}$.

Para países cuyas necesidades y recursos lo permitan, el paso siguiente es la implementación del tamizaje médico genético, con consejo genético, diagnóstico prenatal y servicios asociados para identificación de riesgo y manejo. La Tabla 2 indica los servicios médicos genéticos considerados apropiados para países de bajo y mediano ingreso para pesquisar, diagnosticar o prevenir las consecuencias de algunos defectos de nacimiento ${ }^{23}$.

Prioridades para la atención y la prevención de defectos del nacimiento en países en desarrollo. Se identificaron tres prioridades de acción para colaborar en la iniciación y desarrollo de servicios de atención y prevención de defectos del nacimiento: (A) Apoyar la investigación mantenida para la colección y depuración de los datos sobre defectos del nacimiento; (B) Proveer consejo práctico y apoyo a países interesados en desarrollar servicios médicos genéticos; y (C) Promover el desarrollo de la capacitación del recurso humano y la transferencia tecnológica.

- Prioridad 1: Apoyar la investigación mantenida para la recolección y depuración de datos relevantes para el desarrollo de servicios médicos genéticos. La base de datos del reporte global de la MOD provee estimaciones tipo sobre la prevalencia al nacimiento de defectos congénitos genéticos en 191 países. Las estimaciones del reporte sobre mortalidad infantil difieren apreciablemente de los datos de la OMS sobre muertes infantiles por anomalías congénitas, y estas diferencias no pueden ser explicadas solamente por la diferencia en las definiciones entre defectos del nacimiento $\mathrm{y}$ anomalías congénitas ${ }^{10,24,25}$.

Se acordó que es necesaria la identificación progresiva y recolección de información de 


\section{Tabla 2. Servicios médicos genéticos considerados apropiados para países de bajo y mediano ingreso} para pesquisar, diagnosticar o prevenir las consecuencias de algunos defectos de nacimiento ${ }^{23}$

Preconcepcionales

1. Identificación de portadores utilizando genealogías familiares

2. Identificación de portadores para enfermedades recesivas comunes, hemoglobinopatías (índices, electroforesis, $\mathrm{ADN}$ ) y fibrosis quística (ADN)

Antenatales

3. Negatividad grupo sanguíneo Rhesus

4. Síndrome de Down (edad materna avanzada, suero materno, ultrasonido)

5. Defectos del tubo neural (suero materno y ultrasonido)

6. Malformaciones mayores (búsqueda de anomalías fetales)

7. Identificación de portadores para enfermedades recesivas comunes, hemoglobinopatías Postnatales $(\mathrm{ADN})$ y fibrosis quística (ADN)

8. Tamizaje neonatal (usando tarjetas de Guthrie)

9. Hipotiroidismo congénito

10. Enfermedades de células falciformes

11. Ictericia neonatal/deficiencia de G6PD

12. Errores congénitos del metabolismo

buena calidad en países en desarrollo sobre la prevalencia al nacer de defectos del nacimiento. Debieran hacerse esfuerzos para identificar bases de datos existentes; particular atención debe prestarse a la recolección de datos sobre mortalidad relacionada con defectos del nacimiento, discapacidad y años de vida ajustados a discapacidad (DALYs).

La efectividad de los programas de atención y prevención de defectos del nacimiento es limitada en países en desarrollo. Cuba fue el primer país en desarrollar servicios médicos genéticos extendidos $^{26}$. Sudáfrica, enfrentada a una pandemia de VIH/SIDA, está haciendo progresos hacia este tipo de servicio aprovechando experiencias ganadas de servicios de atención primaria de salud basados en la comunidad en un área rural ${ }^{11,27}$. El éxito de China y Chile en la prevención de defectos del tubo neural con suplementación y fortificación con ácido fólico también están documentados ${ }^{28,29}$.

El peso de los defectos del nacimiento incluye, además de la carga en la prevalencia al nacer, mortalidad y morbilidad, un costo económico para los individuos, las comunidades y los países. Casi no hay información disponible en naciones en desarrollo sobre las implicancias financieras de los defectos de nacimiento. Sin esta información será difícil persuadir a las agencias internacionales y los gobiernos de esas naciones sobre la importancia de desarrollar programas nacionales para la atención y prevención de defectos del nacimiento. Una estrecha interacción con economistas de salud tendiente a desarrollar un mejor conocimiento de los aspectos económicos de los defectos del nacimiento es, por ello, imperativo.

El estudio de los aspectos éticos, legales y sociales (ELSI) de la genética humana ha sido instituido y reflexionado extensamente en naciones industrializadas. Mucho de la discusión de ELSI no es necesariamente aplicable al variado medio social, cultural, religioso y legal de las naciones. ELSI necesita ser integrado con el conocimiento y la experiencia ganados en el desarrollo de servicios médicos genéticos en naciones en desarrollo, en un marco de trabajo más amplio y su discusión.

- Prioridad 2: Proveer consejo práctico y apoyo a países interesados en desarrollar servicios médicos para la atención y prevención de defectos del nacimiento. Los países iniciando y desarrollando servicios genéticos para defectos del nacimiento requieren asistencia. La intención 
de desarrollar e implementar un plan de servicio médico genético nacional utilizando un equipo de trabajo multidisciplinario fue documentada por $\mathrm{HGN}^{12}$.

- Prioridad 3: Apoyar la capacitación de recursos humanos y transferencia tecnológica. Se acordó que es esencial capacitar recursos humanos. Profesionales de la salud -particularmente en atención de salud primaria- deben ser educados y entrenados en los servicios requeridos para la atención y prevención de defectos del nacimiento y existe experiencia de países con los cuales colaborar. Por ejemplo, Chile, Cuba, y Sudáfrica han desarrollado curricula y métodos para entrenar trabajadores en atención de salud primaria en cuidado y prevención de defectos del nacimiento y Chile está actualmente evaluando el uso de telemedicina para este propósito. Sudáfrica, en colaboración con MOD, está haciendo lo mismo.

\section{Meta 4}

Desarrollar un plan a 5 años plazo en la OMS para el fortalecimiento de la atención y prevención de hemoglobinopatías en países de bajo y mediano ingreso. Por los defectos del nacimiento generalmente hay tópicos relacionados con la aplicación de principios éticos desarrollados en un ambiente occidental y cuya aplicación debe considerar las diversas circunstancias sociales, culturales y religiosas de países en desarrollo. Es muy importante tener información sobre los mejores métodos para consejo genético y apoyo social para familias con, por ejemplo, hemoglobinopatías en países con diferentes culturas ${ }^{30,31}$.

\section{Meta 5}

Determinar cómo pudieran contribuir potenciales referentes: Oficinas Regionales de la OMS; agencias gubernamentales internacionales y nacionales; fundaciones y otras organizaciones no gubernamentales; grupos de apoyo de padres/ pacientes; el sector privado; y organizaciones donantes. El progreso requerirá la coordinación de la OMS con otras organizaciones dedicadas al cuidado y prevención de defectos congénitos, como la MOD Birth Defects Foundation, U.S. Centers for Disease Control and Prevention (CDC), International Clearinghouse for Birth Defects Surveillance (ICHBDS), World Alliance of Organiza- tions for the Management and Prevention of Birth Defects (WAO) e International Genetic Alliance (IGA). También es importante la experiencia y compromiso de las organizaciones nacionales de apoyo y consorcios y redes existentes, y las futuras redes norte/sur y sur/sur. Los roles y responsabilidades de este consorcio debieran incluir la apología temática, la transferencia tecnológica, la capacitación, el incentivo de la investigación, la promoción de lineamientos éticos y las finanzas.

Numerosos errores de percepción permiten explicar por qué el cuidado y prevención de los defectos del nacimiento han recibido poca atención de donantes internacionales y agencias de salud. Incluyen una falta de conocimiento de la carga global de defectos congénitos; la creencia errónea de que un cuidado y prevención efectivas requieren intervenciones costosas y de alta tecnología, que están más allá de los presupuestos de salud de los países en desarrollo, y preocupación porque la atención a defectos del nacimiento desviará fondos de otras prioridades de salud materna, del recién nacido y el niño.

Es vitalmente importante que la naturaleza y beneficios de los servicios de genética médica sean promovidas a las oficinas regionales de la OMS, a los que elaboran las políticas de los Ministerios de Salud, a los participantes en salud pública, atención primaria, y especialistas en obstetricia y pediatría. Esto podría lograrse a través de cursos en Genética Comunitaria auspiciados por las Oficinas Regionales de OMS y dirigidos a audiencias específicas y según las necesidades de la población local.

La implementación y el fortalecimiento de los servicios médicos genéticos en países en desarrollo requiere educación profesional, entrenamiento, fondos para laboratorios y otros servicios de apoyo. Hay un considerable potencial en la OMS para apoyar la transferencia tecnológica y capacitación a través de la coordinación, utilizando las estructuras e influencia de sus oficinas regionales.

Hay necesidad de conseguir datos mejores sobre la carga global de los defectos del nacimiento, incluyendo prevalencia al nacer, mortalidad y discapacidad. La vigilancia de defectos del nacimiento es conducida generalmente a nivel local. Existen también escasas redes regionales, por ejemplo, la EUROCAT y redes internacionales 
como la International Clearinghouse for Birth Defects Monitoring Systems (ICBDMS). La OMS y sus entidades asociadas como la MOD pueden jugar un rol importante en identificar, calificar e incorporar datos sobre prevalencia, mortalidad, discapacidad y costos económicos de acción o inacción en relación a defectos del nacimiento.

Casi no hay información disponible de estos países sobre las consecuencias financieras de la acción o inacción en los defectos del nacimiento en general 0 en los costos o beneficios de otras intervenciones como el tamizaje neonatal. Es crítico que la OMS trabaje con organizaciones asociadas, aquellas representadas en la reunión a que alude este documento y otras como el National Institutes of Health o la European Union para estimular dicha evaluación.

Hay dos opciones disponibles para resolver la actual escasez de financiamiento de servicios médicos genéticos para el cuidado y prevención de defectos congénitos. Ambas comprometen el reconocimiento inicial de OMS al más alto nivel la Asamblea Mundial de Salud- de la carga global de los defectos del nacimiento y la necesidad de servicios médicos genéticos en naciones en desarrollo. La voluntad política de apoyo sería un adelanto dado el alto costo total de los defectos del nacimiento y el financiamiento para servicios médicos genéticos estaría más disponible por los gobiernos nacionales. Las organizaciones internacionales de salud estarían más dispuestas a considerar programas con financiamiento para la investigación, cuidado y prevención de los defectos congénitos.

\section{CONCLUSIÓN}

En 1964, como resultado de la transición en salud, el entonces Director General de la OMS reconoció la necesidad del desarrollo de los servicios de

\section{REFERENCIAS}

1. Rose DW. Images of America: March of Dimes. Charleston: Arcadia Pub, 2003.

2. Stevenson AC, Johnston HA, Stewart MIP, Golding DR. Congenital Malformations. A report of a study of a series of consecutive births in 24 countries. genética médica en naciones industrializadas ${ }^{3}$. Veinte y un años después, el grupo asesor del Programa de Enfermedades Hereditarias reconoció que la transición en salud en países en desarrollo, tendría las mismas necesidades de servicios para el cuidado y la prevención de defectos del nacimiento en un futuro cercano y establecieron los principios básicos y perdurables en los cuales estarían basados estos servicios ${ }^{6}$.

Los participantes en la reunión de OMS-MOD convinieron unánimemente en que los servicios para el cuidado y prevención de defectos del nacimiento en general y para hemoglobinopatías en particular, debieran ser una prioridad para la OMS en países en desarrollo. Los pasos requeridos para implementar ambos son similares. Establecer servicios para el cuidado y prevención de defectos del nacimiento en países en desarrollo reducirá la mortalidad y la discapacidad por hemoglobinopatías en todo el mundo. Similarmente, establecer servicios para el cuidado y prevención de hemoglobinopatías en poblaciones de alto riesgo proveerá un marco y la experiencia puede entonces ser aplicada a reducir la carga de otros defectos del nacimiento. Al considerar estas etapas, son dos los principios que deben guiar el desarrollo de las futuras acciones propuestas: Bien común y Salud para Todos.

Los recién nacidos y niños con defectos del nacimiento son un grupo particularmente vulnerable y poco reconocido en naciones en desarrollo. El inicio y desarrollo de servicios para el cuidado de estas poblaciones en riesgo y la implementación de servicios para la prevención de defectos del nacimiento honraría el concepto sobre la importancia de la Salud para Todos. Dado que los niños son también miembros de sus familias y comunidades, el establecimiento de servicios que reduzcan la carga de los defectos del nacimiento en estas poblaciones vulnerables también puede ser vista como un Bien Común.

World Health Organization, Geneva, Switzerland. 1966.

3. World Health Organization. Human genetics and public health. Technical Report Series No. 282. WHO, Geneva, Switzerland. 1964.

4. Christianson AL, Modell B. Medical Genetics in Developing Countries. Ann Rev in Genomics \& 
Hum Genetics 2004; 5: 219-265. [disponible en www.annualreviews.org ]

5. PENCHASZADEH VB. Implementing comprehensive genetic services in developing countries: Latin America. Birth Defects: Original Article Series 1992; 28: 17-26.

6. World Health Organization. Advisory Group. Community approaches to the control of hereditary diseases. WHO, Geneva, Switzerland. 1985.

7. World Health Organization/World Alliance for the Prevention of Birth Defects. Services for the Prevention and Management of Genetic Disorders and Birth Defects in Developing Countries. WHO, Geneva, Switzerland. 1999.

8. Modell B, Kulev A. The history of community genetics: the contribution of the haemoglobin disorders. Community Genetics 1998; 1: 3-11.

9. Penchaszadeh VB. Genetics and Public Health. Bulletin of PAHO 1994; 28: 62-72.

10. Christianson AL, Howson CP, Modeil B. March of Dimes Global Report on Birth Defects: the hidden toll of dying and disabled children. March of Dimes Birth Defects Foundation, White Plains, New York, USA. 2006.[disponible en www.marchofdimes.com]

11. Christianson AL, Venter PA, Modiba JH, Nelson MM. Development of a primary health care clinical genetic service in rural South Africa- The Northern Province experience, 1990-1997. Community Genetics 2000, 3: 77-84.

12. Wordd Health Organization. Primary Health Care Approaches for Prevention and Control of Congenital and Genetic Disorders. WHO, Geneva, Switzerland, 2000.

13. MurRay CJL, LÓpez AD. Global Burden of Disease and Injury Series. Volume I: The Global Burden of Disease. Boston: Harvard School Public Health, 1998.

14. New Shorter Oxford English Dictionary (Volume I). L Brown (Ed). Clarendon Press, Oxford. 1993.

15. CaRmona RH. The global challenge of birth defects and disabilities. Lancet 2005; 366: 1142-4.

16. Wordd Health Organization. International statistical classification of diseases and related health problems. Tenth revision. WHO, Geneva, Switzerland. 1992.

17. Shibuya K, MurRay CJ. Congenital Anomalies. In: Murray CJ, Lopez AD, eds. Global Burden of Disease and Injury Series. Volume III. Health dimensions of sex and reproduction. Boston: Harvard School Public Health, 1998.

18. World Health Organization. Guidelines for the development of national programmes for monitoring birth defects. World Health Organization. 1993.
19. WHO/ICBDMS/EUROCAT. World Atlas of Birth Defects, $1^{\text {st }}$ Edition. Geneva, Switzerland: World Health Organization, 1998 (a).

20. Wordd Health Organization. Control of Hereditary Diseases. WHO Technical Report Series 865. World Health Organization. Geneva, Switzerland. 1996 (a).

21. Czeizel AE, Intôdy Z, Modell B. What proportion of congenital abnormalities can be prevented? BMJ 1993; 306: 499-503.

22. Alwan A, Model B. Community control of genetic and congenital disorders. Eastern Mediterranean Regional Office Technical Publication Series 24. WHO: Regional Office of the Eastern Mediterranean, Alexandria, Egypt. 1997.

23. World Health Organization. Management of birth defects and haemoglobin disorders. Report of a joint WHO-March of Dimes meeting, WHO, Geneva, Switzerland. 2006.

24. World Health Organization. World Health Report 2005. Make every mother and child count. WHO, Geneva, Switzerland. 2005 (a).

25. Bryce J, Boschi-Pinto C, Shibuya K, Biack RE, WHO Child Health Epidemiology ReFerence Group. WHO estimates of the causes of death in children. Lancet 2005; 365: 1147-52.

26. HEREDERO L Comprehensive national genetic program in a developing country-Cuba. Birth Defects Original Article Series 1992; 28: 52-7.

27. National Department of Health (South Africa). National policy guidelines for the management and prevention of congenital disorders, birth defects and disability. National DOH, Pretoria, South Africa. 2001.

28. BerRy RJ, Li Z, ERICKSON JD, Li S, Moore CA, Wang $\mathrm{H}$ ET AL. Prevention of neural-tube defects with folic acid in China. N Engl J Med 1999; 341: 148590.

29. HertrampF E, Cortés F. Folic acid fortification of wheat flour: Chile. Nutrition Rev 2004; 62: S44S49.

30. Weatherall DJ, Akinyanju O, Fucharoen S, Olivieri $\mathrm{N}$, Musgrove P. Inherited Disorders of Hemoglobin. In: Disease Control Priorities in Developing Countries (ed. by D. Jamison \& e. al), pp. 663680. Oxford University Press and the World Bank, New York, Washington. 2006.

31. Weatherall DJ, CiegG JB. The Thalassaemia Syndromes. $4^{\text {th }}$ Edition. Blackwell Science. Oxford, United Kingdom. 2001. 